\title{
MRI Estimation of Liver Function: Comparison Between Normals and Cirrhotic Patients with Different MELD-Na Scores
}

\author{
Numan C. Balci ${ }^{*}$, Shiva Kumar², Terrence Lee St. John ${ }^{3}$, Hidayath Ali Ansari ${ }^{1}$, Ahmad Alduaij ${ }^{4}$ and Antonio D. Pinna ${ }^{5}$
}

*Correspondence: balcin@clevelandclinic abudhabi.ae

\author{
'Cleveland Clinic Abu Dhabi Imaging Institute, UAE. \\ ${ }^{2}$ Cleveland Clinic Abu Dhabi Digestive Disease Institute, UAE. \\ ${ }^{3}$ Cleveland Clinic Abu Dhabi Research Department, UAE. \\ ${ }^{4}$ National Reference Laboratory, UAE. \\ ${ }^{5}$ Abdominal Transplant Center, Cleveland Clinic Florida, Weston, USA.
}

\begin{abstract}
Purpose: Currently used prognostic models in cirrhosis rely on clinical and laboratory criteria. Quantitative MRI estimation of liver function could determine degree of hepatic synthetic function impairment and could consequently be used as a prognostic tool in patients with cirrhosis. Our aim was to estimate liver function using MRI and assess its correlation with MELD-Na score, an established laboratory-based prognostic model in cirrhosis.

Methods: 52 subjects without liver disease (controls) and 79 patients with cirrhosis were retrospectively evaluated. Patients with cirrhosis were stratified based on MELD-Na scores into the following groups: 6-10, 11-17, 18-24 and 25-40. All subjects underwent MRI with hepatobiliary contrast agent Gadoxetic acid (Gd$\mathrm{EOB}$ ) and T1 relaxation time map of all subjects were created for pre and postcontrast images. The T1 time ratio between pre- and postcontrast hepatobiliary phase images were determined in all study subjects and correlated with MELD-Na scores in patients with cirrhosis.

$\underline{\text { Results: }}$ The average $\mathrm{T} 1 \mathrm{ratio}$ in healthy controls was 0.83 . The average T1 ratios in patients with MELDNa scores of 6-10, 11-17, 18-24 and 25-40 were 0.79, 0.69, 0.58 and 0.50 respectively. There was significant difference between $\mathrm{T} 1 \mathrm{ratios}$ in controls without liver disease and patients with cirrhosis $(\mathrm{p}<0.001)$. Moreover, T1 ratios among patients with cirrhosis increased with each successive range of MELD-Na scores $(\mathrm{p}<0.001)$.

Conclusion: The T1 map MR estimation of liver function with the use of hepatobiliary contrast agent correlates well with established prognostic models in cirrhosis and could be a valuable adjunct to currently available clinical tools to estimate liver function and thereby enable prognostication in patients with cirrhosis.
\end{abstract}

Keywords: Liver, Function, MRI, Cirrhosis, Hepatobiliary Contrast

\section{Introduction}

The primary determinant of outcome in cirrhosis is the degree of hepatic synthetic function impairment. Estimation of liver functional reserve could also help to predict the success of liver resection including living donor related liver transplantations $[1,2]$. Currently used prognostic models in cirrhosis rely on clinical and laboratory data. The Child-Turcotte-Pugh score has been used to estimate the liver functional status in patients with cirrhosis for the past five decades but relies on clinical criteria that are subject to inter-observer variation. Developed almost two decades ago and utilizing three common objective laboratory parameters, the MELD score (Model for End-stage Liver Disease) score has been extensively validated as a prognostic model in patients with cirrhosis [3]. This model was modified subsequently with the addition of serum sodium level and the MELD-Na score is currently used to stratify patients on the liver 
transplant wait-list in the United States [4].

Apart from clinical and laboratory assessment, there are established and emerging techniques for liver function estimation, that have either been validated and or are under investigation. The Indocyanine green retention (ICG) test can estimate liver function by using a water-soluble anionic compound that is taken up by hepatocytes and excreted to into the bile. It is used mainly in surgical candidates to estimate liver functional reserve. However, this test does not provide anatomical detail for regional differences of liver function [5]. The nuclear medicine-based tests use radiotracers that either have hepatobiliary pathway ( ${ }^{99 \mathrm{~m} T c-G S A}$ and ${ }^{99 \mathrm{~m} T c-m e b r o f e n i n)}$ or rely on Kupffer cell uptake when ${ }^{99 \mathrm{~m}} \mathrm{Tc}$-sulfur colloid is used. Although these tests have been shown to be reliable, the level of anatomical detail for surgical planning is not as accurate as cross-sectional imaging techniques, necessitating additional investigations which may entail additional radiation exposure [6]. The use of hepatobiliary contrast agents in liver imaging has enabled the estimation of liver function by the degree of hepatobiliary contrast uptake. The T1 relaxation time is reduced proportional to the hepatic uptake of the agent and can be quantitatively analyzed on MRI. Furthermore, this is not an additional investigation for the patient and could be performed during the initial imaging evaluation in patients with liver disease, without need for additional testing [7]. The anatomical detail of MRI also enables one step examination of focal liver lesions, surgical planning and liver function [6]. The double dose approach has been proposed for the use of hepatobiliary contrast agent Gadoxetic acid to achieve an improved T1 relaxation time shortening for the assessment of HCC in cirrhotic patients [8]. The aim of this study is to retrospectively evaluate the role of Gadoxetic acid in the estimation of liver function and correlate this with MELD Na scores in patients with liver cirrhosis.

\section{Materials and methods}

This retrospective study was approved by the institutional review board and informed consent was waived.

\section{Subjects}

We first performed a retrospective chart review of all patients who underwent liver MRI between May 2018 till May 2020 with the use of hepatobiliary contrast agent Gadoxetic acid (Gd-EOB-DTPA). Patients were divided into two cohorts - those with no history of chronic liver disease and normal clinical laboratory findings and those with clinically established cirrhosis. The normal group consisted of 52 (20 male, 32 female ages 20-63, mean age 46.9) patients who underwent MRI of the liver with and inconclusive ultrasound examination for further imaging $(n=28)$, and patients who were donor candidates for living related liver transplantation and also underwent liver biopsy for fat quantification $(n=11)$. Patients with cirrhosis were diagnosed clinically, based on only imaging findings $(n=11)$, endoscopic evaluation of varices together with imaging findings $(n=41)$ and histopathology $(n=27)$. Patients with liver cirrhosis underwent liver MRI for surveillance for HCC. The total number of patients with liver cirrhosis were 79 (43 male, 34 female ages 19-78, mean 55.7). The MELD-Na score of the cirrhotic patients were calculated according to formula: MELD-Na score=MELDscore-SerumNa$(0.025 *$ MELDscore*(140-SerumNa)) + 140 (10). All MELD-Na scores were calculated with the laboratory values from charts using MD+CALC free online portal.

The patients were stratified into four groups according to their MELD-Na scores. The group 1 (MELD score 6-10), group 2 (MELD score 11-17) group 3 (MELD score 18-24), group 4 (MELD score 25-40). 15 patients with liver cirrhosis had followup liver MRI with hepatobiliary contrast agent at an interval greater than 6 months. In this subset,interval MELD-Na score changes and $\mathrm{T} 1$ ratio changes on MRI were reviewed separately. The interval between MELD-Na score and liver MRI was within less than 10 days in all patients.

\section{MRI acquisition}

All patients underwent MRI of the liver using a 3.0 Tesla MRI (MagnetomSkyra; Siemens Healthcare, Erlangen,Germany) with an eight-channel body phased-array coil. The MR protocol consisted of breath-hold transverse T1-weighted in- and out-of-phase two-dimensional gradient echo sequences (TR/ in phase TE, 220ms/2.6 ms; out-of-phase TE, $1.5 \mathrm{~ms}$; flip angle, $65^{\circ}$;field of view, $400 \times 300 \mathrm{~mm}$; matrix, 256x115; section thickness, $6 \mathrm{~mm}$ ), T2-weighted half Fourier acquisition single shot turbo spin echo sequences (TR/TE, 600ms/91 ms; flip angle, $138^{\circ}$ field of view, $400 \times 300$ mm; matrix, 384 x173; slice thickness, $6 \mathrm{~mm}$ ) in axial and coronal planes. A dose of $0.2 \mathrm{~mL} / \mathrm{kg}$ $(0.050 \mathrm{mmol} / \mathrm{kg}) \mathrm{Gd}$-EOB-DTPA (Eovist, Bayer Pharma) was administered intravenously at $1.0 \mathrm{~mL} / \mathrm{s}$ for the dynamic study, followed by a $30-\mathrm{mL}$ saline flush. Contrast-enhanced MRI was performed using an axial fat-suppressed three-dimensional T1-weighted spoiled gradient echo sequence (TR/TE, 3.1/1.2; flip angle, $11.5^{\circ}$; field of view, $380 \times 280$ mm; matrix, 374x175; section thickness, $2 \mathrm{~mm}$ ), Gd-EOB-DTPA-enhanced hepatocytephase images were obtained 20 min after contrast agent administration. T1 mappingsequence was acquired using a dual flip angle 3D gradient echo technique beforeand 20 minutes after contrast injection during hepatobiliary phase with the use of Siemens Maplt software for all the patients. Imaging parameters were: 48 axial slices of $4 \mathrm{~mm}$ thickness acquired within an 18-second breath-hold with repetition time (TR) of $3.87 \mathrm{msec}$, echo time (TE) of $1.32 \mathrm{msec}$, flip angle of $2^{\circ}$ and $13^{\circ}$, acquisition matrix of $320 \times 168$, parallel imaging (Siemens GRAPPA) factor of 2, field of view of $300 \times 400 \mathrm{~mm}$, and bandwidth of $446 \mathrm{~Hz} /$ pixel.

\section{Image Analysis}

All measurements were performed by an experienced abdominal radiologist who had 25 years of experience in abdominal MRI. SyngoVia (Siemens Healthcare, Erlangen, Germany) 
workstation was used for measurements. The T1map images before and after hepatobiliary contrast were uploaded to SyngoVia. Same size region of interest (ROI) was used for every measurement. Each liver segment was measured separately, a total of nine liver segment measurements were performed for each liver on T1 map images before contrast injection and on 20 minute postcontrast images. The T1 ratio between precontrast and 20 minute postcontrast images were calculated as $\mathrm{T} 1$ ratio=T1 time precontrast-T1 time postcontrast)/ T1 time precontrast.

\section{Statistical Analysis}

The difference between the average $\mathrm{T} 1$ ratios in normal subjects and in cirrhotic livers with different MELD-Na scores was examined using an OLS regression where normal subjects served as the reference group, and each MELD group was dummy coded to examine its contrast with the normal group. A two-tailed alpha criteria of .05 was used to determine statistical significance.

\section{Results}

The average $T 1$ ratio in normal subjects without liver disease was 0.827 (Figure 1). In patients with cirrhosis, the average T1 ratios in the four MELD-Na groups were: 0.79 (Group 1; $\mathrm{n}=29$ patients), 0.69 (Group 2; n=33), 0.58 (Group 3; $n=14$ ) and 0.50 (Group 4; $n=3$ ) (Figure 2). There were significant differences between the average $T 1$ ratios of normal patients and in four different MELD-Na groups. There was significant difference between $\mathrm{T} 1$ ratios in controls without liver disease and

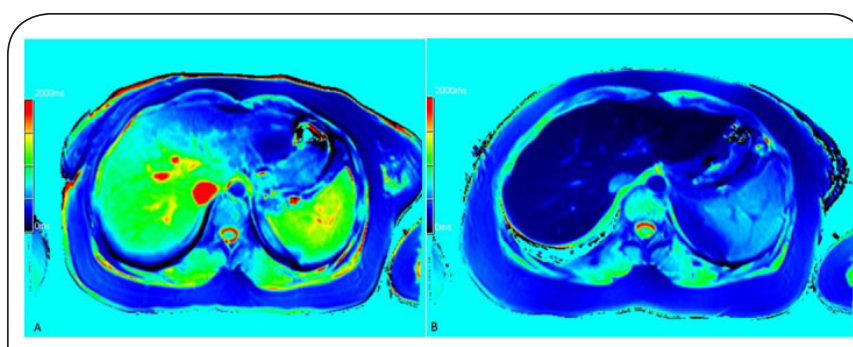

Figure 1. T1 map of a normal subject. The avararege T1 values are displayed on color mapped precontrast (a) and 20 minpostcontrast (b) images.

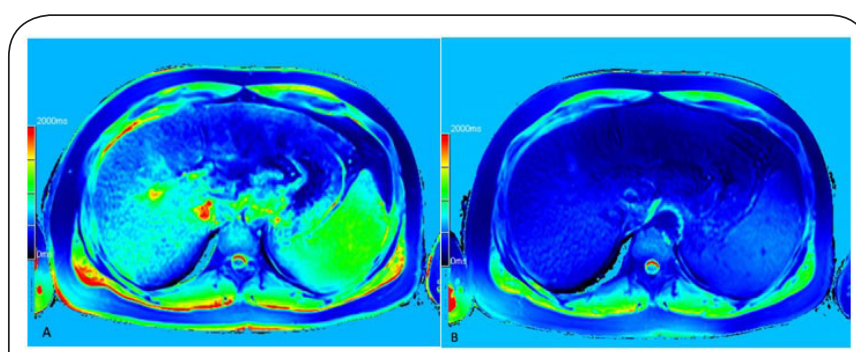

Figure 2. T1 map of a cirrhoticliver (MELD-Nascore 18). The avararege $\mathrm{T} 1$ values are displayed on color mapped precontrast (a) and 20 minpostcontrast (b) images. patients with cirrhosis $(p<0.001)$. Moreover, T1 ratios among patients with cirrhosis increased with each successive range of MELD-Na scores $(p<0.001)$ (Table 1$)$.

The average T1 ratios of different MELD-Na groups were listed on the Table 1.

Table 1. MRI T1 ratio in Normal and in Cirrhotic Patients with different MELD-Na scores.

\begin{tabular}{llllll}
\hline & $\begin{array}{l}\text { Number } \\
\text { of } \\
\text { Patients }\end{array}$ & $\begin{array}{l}\text { Average } \\
\text { T1 Ratio }\end{array}$ & $\begin{array}{l}\text { Regression } \\
\text { Coef. }\end{array}$ & t-value & p-value \\
\hline Normal & 52 & 0.83 & 0.83 & 157.06 & $<0.001$ \\
MELD-Na 06-10 & 29 & 0.79 & -0.03 & -3.55 & 0.001 \\
MELD-Na 11-17 & 33 & 0.69 & -0.13 & -15.58 & $<0.001$ \\
MELD-Na 18-24 & 14 & 0.58 & -0.24 & -21.30 & $<0.001$ \\
MELD-Na 25-30 & 3 & 0.50 & -0.33 & -14.49 & $<0.001$ \\
\hline
\end{tabular}

23 cirrhotic patients were on follow-up. The patients on follow-up revealed either stable $(n=3)$, decreased $(n=4)$ and increased ( $n=16)$ MELD-Na scores. One out of three patients with stable MELD-Na scores had stable T1 ratios, three out of four patients with decreased MELD-Na score had increased $\mathrm{T} 1$ ratio, one patient had slightly increased $\mathrm{T} 1$ ratio. All of the 16 patients with increased MELD-Na score had decreased T1 ratio. The number of follow-up observations were too small to make a statistical analysis.

\section{Discussion}

The results of our study have revealed that the estimation liver function using MRI corelates with the MELD-Na score and there is statistically significant difference between the MRI estimated liver function of normal and cirrhotic livers. Any chronic insult to the liver parenchyma may result in hepatocellular injury that later leads to development of fibrosis, with continued progression resulting in cirrhosis and consequently hepatic synthetic function impairment [8]. There are several scoring systems for the clinical estimation of hepatic synthetic function in cirrhotic patients. Child-Pugh, MELD and MELD-Na scores are the methods used in clinical practice. However, all these scoring systems have limitations, and these prognostic models have limited utility in patients with compensated cirrhosis. The Child-Pugh score includes assessment of albumin, bilirubin, prothrombin time international normalized ratio (INR), ascites and hepatic encephalopathy. However, this scoring system has challenges due to its limited discriminatory ability and clinical parameter subjectivity. The MELD score uses more objective variables that include bilirubin, INR, and creatinine and has been widely used in prediction of the mortality of patients with cirrhosis. However, MELD score has also been noted to under-estimate disease severity in subsets of patients with decompensated cirrhosis. In the meantime, MELD-Na is more widely used due to its enhanced prognostic accuracy. 
Balci et al., Medical Imaging and Radiology 2021,

http://www.hoajonline.com/journals/pdf/2054-1945-9-1.pdf

doi: $10.7243 / 2054-1945-9-1$

MELD-Na scoring system has been successfully used to classify the severity of cirrhosis and is a decision-making tool for the management of cirrhotic patients including liver transplantation [11-13]. The MR estimated liver function based on $\mathrm{T} 1$ ratio was significantly different between the different severity of MELD-Na groups. Our results are similar to previously published articles that have shown the correlation of estimated liver function with MELD score. In our study we used the MELD-Na score, which has been shown to reflect the functional status of the liver better than MELD score [15].

The MR estimation of liver function relies on the degree of hepatobiliary contrast uptake in the liver. Gadoxetic acid $(\mathrm{Gd}-\mathrm{EOB})$ is absorbed by the hepatocytes via organic aniontransporting polypeptides (OATP) $1 \mathrm{~B} 1$ and $1 \mathrm{~B} 3$ and is then eliminated in bile via multidrug resistance protein (MRP). Gd-EOB reduces the $\mathrm{T1}$ timein MRI and has been used for liver imaging especially increasing sensitivity and specificity in HCC screening. The agent has extracellular and hepatobiliary distribution after injection which makes it a double agent. The $\mathrm{T} 1$ relaxation time is further reduced with the increase of the injection dose and investigations have shown better delineation of HCC in arterial phase when double dose is used [9,16-18]. The effect of double dose Gd-EOB has not been investigated in hepatobiliary phase to estimate the liver function. Our results have shown that the $\mathrm{T} 1$ relaxation time has dropped more than the prior studies [16]. The T1 relaxation time measurement is a more accurate method than signal intensity measurement during hepatobiliary phase imaging, we used the variable flip angle method and created a T1 map for the entire liver to estimate the liver function in each segment by measuring the $\mathrm{T} 1$ values on map images. The T1 map images provided the anatomical detail to better delineate liver segments in our study.

We have also evaluated the follow-up results of MELD-Na scores in patients with liver cirrhosis and compared them with the MRI estimated liver function [19]. The MELD-Na scores may change over time in the patients with liver cirrhosis. Most of the time the score worsens and in few instances the score may get better with the antiviral therapy. In our dataset, we had 23 patients on follow-up whose MELD-Na scores and estimated liver function on MRI correlated. The number of patients were not enough to determine the statistical significance.

The main limitation of this study is the lack of ICG test as reference of standard to correlate with MRI estimated liver function. The ICG test provides a comprehensive evaluation of hepatic function with the degree of uptake, metabolism, and excretion, and is commonly used for the preoperative evaluation of liver function [20]. Nevertheless, the differences in T1 ratios between different MELD-Na score patient populations and the differences between $\mathrm{T} 1$ ratios of normal and cirrhotic patient population were significant.

In conclusion, our review of T1 relaxation time ratios differed among patients with and without cirrhosis and increased across the range of MELD-Na scores. Adding T1 relaxation time measurements in hepatobiliary contrast enhanced MRI examination may both screen for HCC and determine the functional status of the liver in cirrhotic patients. Future studies are needed to investigate, if the MRI estimation of liver function may predict the outcome of patients with liver cirrhosis.

\section{Competing interests}

The authors declare that they have no competing interests.

Authors' contributions

\begin{tabular}{|l|c|c|c|c|c|c|}
\hline Authors' contributions & NCB & SK & TLS & HAA & AA & TP \\
\hline Research concept and design & $\sqrt{ }$ & $\sqrt{ }$ & -- & -- & -- & $\sqrt{ }$ \\
\hline Collection and/or assembly of data & $\sqrt{ }$ & -- & -- & $\sqrt{ }$ & $\sqrt{ }$ & $\sqrt{ }$ \\
\hline Data analysis and interpretation & $\sqrt{ }$ & -- & $\sqrt{ }$ & -- & -- & -- \\
\hline Writing the article & $\sqrt{ }$ & $\sqrt{ }$ & -- & -- & -- & -- \\
\hline Critical revision of the article & $\sqrt{ }$ & $\sqrt{ }$ & -- & -- & -- & -- \\
\hline Final approval of article & $\sqrt{ }$ & $\sqrt{ }$ & -- & -- & -- & -- \\
\hline Statistical analysis & -- & -- & $\sqrt{ }$ & -- & -- & -- \\
\hline
\end{tabular}

\section{Acknowledgments}

Research reported in this publication was supported by the Mubadala Grant Award 2019. The content is solely the responsibility of the authors.

\section{Publication history}

Senior Editor: Domenico Rubello, Santa Maria della Misericordia Hospital, Italy.

Received: 29-May-2021 Final Revised: 26-Jun-2021

Accepted: 23-Aug-2021 Published: 15-Sept-2021

\section{References}

1. Jalan R, Saliba F, Pavesi M, Amoros A, Moreau R, Gines P, et al. Development and validation of a prognostic score to predict mortality in patients with acute-on-chronic liver failure. J Hepatol. (2014) 61:103847.

2. Wagener G. Assessment of hepatic function, operative candidacy, and medical management after liver resection in the patient with underlying liver disease. Semin Liver Dis. (2013) 33:204-12.

3. Kamath PS, Wiesner RH, Malinchoc M et al. A model to predict survival in patients with end-stage liver disease. Hepatology. 2001 Feb;33(2):46470).

4. Kim WR, Biggins SW, Kremers WK et al. Hyponatremia and mortality among patients on the liver-transplant waiting list. N Engl J Med. 2008 Sep 4;359(10):1018-26

5. Kitano S, Kim YI. ICG clearance in assessing cirrhotic patients with hepatocellular carcinoma for major hepatic resection. HPB Surg.1997;10(3):182-3.

6. Matesan MM, Bowen SR, Chapman TR, Miyaoka RS, Velez JW, Wanner MF, Nyflot MJ, Apisarnthanarax S, Vesselle HJ. Assessment of functional liver reserve: old andnew in 99mTc-sulfur colloid scintigraphy. Nucl Med Commun. 2017 Jul;38(7):577-586.

7. Geisel D, Lüdemann L, Hamm B, Denecke T. Imaging-Based Liver Function Tests--Past, Present and Future. Rofo. 2015 Oct;187(10):863-71.

8. Duan T, Jiang H, Xia C, Chen J, Cao L, Ye Z, Wei Y, Song B, Lee JM. Assessing Liver Function in Liver Tumors Patients: The Performance of T1 Mapping and Residual Liver Volume on Gd-EOBDTPA-Enhanced MRI. Front Med (Lausanne). 2020 May28;7:215.

9. Motosugi U, Ichikawa T, Sano K, Sou H, Onohara K, Muhi A, Kitamura T, AmemiyaF, Enomoto N, Matsuda M, Asakawa M, Fujii H, Araki T. Doubledose gadoxeticAcid-enhanced magnetic resonance imaging in patients with chronic liver disease.Invest Radiol. 2011 Feb;46(2):141-5. 
Balci et al., Medical Imaging and Radiology 2021,

10. Kim WR, Biggins SW, Kremers WK et al. Hyponatremia and mortality among patients on the liver-transplant waiting list. N Engl J Med. 2008 Sep 4;359(10):1018-26.

11. Smith A, Baumgartner K, Bositis C. Cirrhosis: Diagnosis and Management. Am Fam Physician. 2019 Dec 15;100(12):759-770.

12. Somsouk M, Guy J, Biggins SW, Vittinghoff E, Kohn MA,Inadomi JM. Ascites improves upon serum sodiumplus model for end-stage liver disease (MELD) for predicting mortality in patients with advanced liver disease.Aliment Pharmacol Ther. 2009;30:741-8.

13. Freitas ACT, Rampim AT, Nunes CP, Coelho JCU. Impact of MELD Sodium on Liver Transplantation waiting list. Arq Bras Cir Dig. 2019 Dec 9;32(3):e1460.

14. Cheng XP, Zhao J, Chen Y, Meng FK, Xu B, Yu HW, Meng QH, Liu YM, Zhang SB, Meng S, Zhang JY, Zhang JY, Duan ZP, Zheng SJ. Comparison of the ability of thePDD-ICG clearance test, CTP, MELD, and MELD-Na to predict short-term and medium-term mortality in patients with decompensated hepatitis B cirrhosis. Eur JGastroenterol Hepatol. 2016 Apr;28(4):444-8.

15. Leise $M$, Cárdenas A. Hyponatremia in Cirrhosis: Implications for Liver Transplantation. Liver Transpl. 2018 Nov;24(11):1612-1621.

16. Marroni CP, de Mello Brandão AB, Hennigen AW, Marroni C, Zanotelli ML, Cantisani G, Fuchs SC; Liver Transplantation Group. MELD scores with incorporation of serum sodium and death prediction in cirrhotic patients on the waiting list for liver transplantation: a single center experience in southern Brazil. Clin Transplant. 2012 Jul-Aug;26(4):E395-401.

17. Haimerl M, Verloh N, Zeman F, Fellner C, Nickel D, Lang SA, Teufel A, Stroszczynski C, Wiggermann P. Gd-EOB-DTPA-enhanced MRI forevaluation of liverfunction: Comparisonbetweensignal-intensitybasedindicesand T1 relaxometry. SciRep. 2017 Mar 7;7:43347.

18. Balci NC, Semelka RC. Contrast agents for MR imaging of the liver. Radiol Clin North Am. 2005 Sep;43(5):887-98.

19. Saito K, Ledsam J, Sourbron S, Hashimoto T, Araki Y, Akata S, Tokuuye K. Measuring hepatic functional reserve using low temporal resolution Gd-EOB- DTPAdynamic contrast-enhanced MRI: a preliminary study comparing galactosyl humanserum albumin scintigraphy with indocyanine green retention. Eur Radiol. 2014Jan;24(1):112-9.

20. Yip TC, Chan HL, Tse YK, Lam KL, Lui GC, Wong VW, Wong GL. OnTreatment Improvement of MELD Score Reduces Death and Hepatic Events in Patients With Hepatitis B-Related Cirrhosis. Am J Gastroenterol. 2018 Nov;113(11):1629-1638.

21. Kokudo T, Hasegawa K, Kokudo N. Assessment of preoperative liver function based on indocyanine green clearance. Hepatology. 2017 Aug;66(2):675-676.

\section{Citation:}

Balci NC, Kumar S, St. John TL, Ansari HA, Alduaij A and Pinna AD. MRI Estimation of Liver Function: Comparison Between Normals and Cirrhotic Patients with Different MELD-Na Scores. Med Imaging Radiol. 2021; 9:1. http://dx.doi.org/10.7243/2054-1945-9-1 